\title{
« Für die war ich Luft »
}

Raconter le corps masculin juif allemand

"Für die war ich Luft". Narrative des männlichen deutsch-jüdischen Körpers

"Für die war ich Luft". Narratives of the Male German Jewish Body

\section{Patrick Farges}

\section{OpenEdition \\ Journals}

Édition électronique

URL : http://journals.openedition.org/ceg/3877

DOI : $10.4000 /$ ceg.3877

ISSN : 2605-8359

\section{Éditeur}

Presses Universitaires de Provence

\section{Édition imprimée}

Date de publication : 15 novembre 2018

Pagination : 157-172

ISBN : 979-10-320-0183-7

ISSN : 0751-4239

Référence électronique

Patrick Farges, « «Für die war ich Luft » », Cahiers d'Études Germaniques [En ligne], 75| 2018, mis en ligne le 25 avril 2020, consulté le 27 novembre 2020. URL : http://journals.openedition.org/ceg/3877 ; DOI : https://doi.org/10.4000/ceg.3877 


\title{
"Für die war ich Luft " \\ Raconter le corps masculin juif allemand
}

\author{
Patrick FARGES \\ Université Paris-Diderot (USPC)/ EA 337 (ICT : Identités - Cultures - Territoires)
}

Dans la tradition marxiste, le matérialisme est inscrit au cœur des rapports sociaux $^{1}$. Les voix/ voies du matérialisme sont multiples lorsqu'on les croise avec le genre. Si l'on considère le genre comme l'ensemble des rapports sociaux de sexe, il est pertinent d'en interroger la matérialité ${ }^{2}$, c'est-à-dire l'ensemble des mécanismes qui font exister le genre dans la tension entre la réalité et les mots pour la dire. Les réflexions actuelles autour de l'" intersectionnalité ${ }^{3}$ " réinterrogent les rapports sociaux à l'aune de la matérialité, afin de saisir divers rapports de pouvoir (race/ classe/ genre). Judith Butler, partant de la scène althussérienne de l'interpellation, développe une réflexion sur la performativité de genre opérée par le langage. Selon Louis Althusser en effet, le langage est une pratique par laquelle l'idéologie 's'adresse' aux individus pour les " recruter ". La performativité butlérienne désigne le processus qui produit des identités socio-genrées, c'est-à-dire un ensemble d'actes performatifs ritualisés qui " naturalise " (voire matérialise) les catégories de genre 4 . Par cette performativité, le corps devient le réceptacle d'un processus de répétition/ stylisation qui incarne les rapports sociaux de genre. L'un des aspects radicaux de cette interpellation est l'injure (sexiste, homophobe, raciste, antisémite). Comme le rappelle Didier Eribon, l'injure préexiste aux individus et participe de leur constitution : elle " me dit ce que je suis dans la mesure même où elle me fait être ce que je suis ${ }^{5}$ ». Opérant par hiérarchisation et généralisation, l'injure fonctionne " fondamentalement comme un rappel à l'ordre ${ }^{6}$ ». Comme toute forme de violence, elle participe de la (re)production de l'ordre social.

1. Voir Louis Althusser, "Du matérialisme aléatoire " [1986], in Multitudes 21, 2005, p. 179-194.

2. Voir Maxime Cervulle, Nelly Quemener, Florian Vörös (dir.), Matérialismes, culture \& communication, t. II " Cultural Studies, théories féministes et décoloniales ", Paris, Presses des Mines, 2016.

3. Kimberlé W. Crenshaw, "Cartographies des marges : intersectionnalité, politique de l'identité et violences contre les femmes de couleur " [1994], trad. O. Bonis, Cahiers du Genre 39, 2005, p. 51-82.

4. Judith Butler, Trouble dans le genre : le féminisme et la subversion de l'identité, trad. C. Kraus, Paris, La Découverte, 2005.

5. Didier Eribon, Réflexions sur la question gay, Paris, Flammarion, 1999, p. 28.

6. Ibid., p. 102. 
Critiquée pendant un temps pour la radicalité de son approche linguistique, Judith Butler a, dans Ces corps qui comptent, voulu retisser les liens entre matérialité et corps, afin de penser les souffrances ainsi que les expériences vécues des corps stigmatisés par les discours de haine ${ }^{7}$. Elle développe ainsi une théorie de l'incorporation', ce processus à travers lequel les normes, contraintes et hiérarchies sociales s'inscrivent dans les corps. Cette notion a partie liée avec les travaux de Pierre Bourdieu sur l'habitus et sur son extériorisation corporelle, l'hexis ${ }^{8}$. Les expériences que vivent les individus en tant qu'êtres sociaux modifient concrètement leur corps, dans sa matérialité, son allure et ses potentialités. Ce qui est incorporé, ce sont des habitudes corporelles, mais aussi des goûts, des schèmes de perception, des catégories morales, des façons d'agir, des manières de voir et de percevoir le monde ${ }^{9}$. L'inscription physique du genre et des rapports de domination passe donc aussi par l'acquisition d'habitudes corporelles en fonction des normes et possibilités disponibles dans l'espace social.

La montée d'un antisémitisme radical en Allemagne a été vécue par les jeunes Juifs comme une atteinte aux représentations corporelles et à l'identité de genre. Ce sont en particulier les enjeux de masculinité qui nous intéressent ici. La masculinité juive allemande en devenir a été bouleversée par les discours, injures et brimades antisémites, inscrites à même les corps. C'est en effet la matérialité des corps - la corporéité - qui en a été affectée : dans les postures, les gestes, les manières d'être et de parler, dans l'habitus corporel. Cette identité blessée s'est racontée a posteriori, dans les narrations autobiographiques et les récits de vie, notamment dans les entretiens d'histoire orale du "Israel-Korpus ${ }^{10}$ ", qui portent les traces de l'injure raciale et genrée. Il s'agira donc de s'intéresser à l'aspect relationnel, voire feuilleté, de la masculinité, ainsi qu'à son aspect intersectionnel. La masculinité est 'relationnelle' parce qu'elle est non seulement produite en interaction avec la féminité, mais aussi parce que les différentes incarnations de masculinité se hiérarchisent les unes par rapport aux autres, et ce dès le plus jeune âge. On peut considérer que les (jeunes) hommes sont tout à la fois les vecteurs d'une masculinité plutôt dominante et qu'ils sont eux-mêmes soumis à ce système de domination. Ils peuvent être dominants dans un contexte et selon un axe de domination donné, et dans le même temps être dominés, en fonction des rapports sociaux de classe ou des rapports sociaux de race : c'est le cas des Juifs dans le contexte d'une montée de l'antisémitisme dans les années 1920 et 1930.

7. Judith Butler, Ces corps qui comptent. De la matérialité et des limites discursives du " sexe ", trad. C. Nordmann, Paris, Éd. Amsterdam, 2009.

8. Pierre Bourdieu, Esquisse d'une théorie de la pratique, précédé de Trois études d'ethnologie kabyle, Genève, Droz, 1972 ; Pierre Bourdieu, La Distinction. Critique sociale du jugement, Paris, Minuit, 1979.

9. Voir Martine Court, "Incorporation ", in Juliette Rennes (dir.), Encyclopédie critique du genre, Paris, La Découverte, 2016, p. 321-330.

10. Voir la présentation du corpus sur le site de l'Institut für deutsche Sprache - Mannheim : [http:// agd.ids-mannheim.de/IS--_extern.shtml], dernière consultation le 18 juillet 2017. 


\section{Les fabriques du masculin}

Pour les jeunes hommes juifs ayant grandi dans l'Allemagne de l'entredeux-guerres, " devenir un homme " était un processus identitaire complexe mettant en jeu des rapports sociaux imbriqués de race, de classe et de genre. Le fait d'être juif produisait des injonctions corporelles et genrées. En fonction de l'appartenance de classe, il fallait " se tenir " d'une certaine manière, agir conformément aux attentes ; en tant que Juif, il fallait faire la démonstration de son appartenance à la nation ou à un milieu social donné. Cela signifiait notamment être capable de se défendre en "bon Allemand ", selon un système de valeurs hérité de la construction nationale et ravivé par la Grande Guerre. Il s'agissait également de faire mentir le vieux stigmate antisémite de l'efféminement. Ce processus était lié à des émotions et affects dont les sources consultées pour la présente étude - entretiens d'histoire orale, récits autobiographiques, correspondances - gardent les traces narratives. Les dynamiques et injonctions de genre inscrites dans les corps des jeunes hommes à l'époque se traduisent ainsi par des récits rétrospectifs de moments significatifs marqués d'affects.

Dans son récit de vie, Paul Alsberg, né en 1919, se souvient d'un moment important de sa scolarité : il insiste sur les interactions entre garçons au sein de la cour d'école, le lieu où - en parallèle à l'instruction dispensée en classe - on apprenait à devenir "quelqu'un ". Apprendre à s'imposer sur ce terrain, c'était aussi apprendre les règles d'un jeu social hiérarchisé reposant sur de fortes injonctions à se comporter " en homme ". Il rappelle l'évidence avec laquelle se produisaient ces interactions :

Ich war der kleinste der gesamten Schule bis zur Quarta. Und natürlich wurde ich hier und da angerempelt und verhauen. Als ich dann zuhause sagte, dass ich mich wehren müsste und nicht kann, weil ich so klein bin, hat mein Vater gesagt : " Ach, da gibt es was " und hat mir Jojitschu-Unterricht geben lassen. Und das nächste Mal, als mich jemand anbaggerte, bevor er sich noch versah, lag er schon auf der Erde. Und danach bin ich immer in Ruhe gelassen worden ${ }^{11}$.

Savoir être un homme, i.e. agir et se comporter conformément à des attentes sociales et genrées, s'apprenait. De même, devenir " allemand ", " juif " voire " juif allemand " était lié à une socialisation et des sociabilités particulières.

Ces instances de sociabilité fonctionnaient comme autant de "fabriques " du masculin ${ }^{12}$, qu'il s'agisse de l'armée, de l'école, de la rue, de la corporation étudiante, de l'association de tir ou de l'association sportive. Au sein de ces lieux sociaux structurés par la compétition, où l'identité se forgeait dans l'interaction avec les pairs, par le truchement de rites d'initiation, d'un esprit de corps, de jeux

11. Paul Alsberg, "Kurzbiografie ", éd. F. Kempf, mars 2006, [http://www.irgun-jeckes.org/?Categor yID=345ctArticleID=744], dernière consultation le 21 avril 2014.

12. L'anthropologue Maurice Godelier évoque les " maisons-des-hommes " chez les Baruyas de Nouvelle-Guinée. Il s'agit d'espaces monosexués où se transmettent, à l'abri des femmes, les composantes de l'hégémonie masculine. Maurice Godelier, La Production des Grands Hommes [1982], Paris, Fayard, 1996. 
de pouvoir, de tensions voire de décharges de violence, des formes de masculinité étaient constamment produites et transmises. La masculinité se fabriquait dans l'entre-soi et par la mise à distance du féminin. Loin d'être anodines, les frictions faisaient partie d'un système complexe. Le corps, vecteur et réceptacle de ces frictions, y jouait un rôle central pour asseoir une position dans la hiérarchie genrée, et donc sociale. La virilité comme idéal de comportement masculin se démontrait et se défendait ; en retour, la masculinité pouvait aussi être blessée. Pour un garçon ou un jeune homme juif, il était d'autant plus important de montrer qu'il était un homme que la stigmatisation antisémite insistait sur la faiblesse supposée des corps juifs masculins, sur leur féminité voire leur efféminement ${ }^{13}$. Si diverses stratégies ont été suivies dans la constitution d'une nouvelle masculinité juive, elles ont convergé pour la faire reposer sur des valeurs de santé, de force et de défense (Wehrhaftigkeit). On constate ainsi la mise en place de pratiques sociales de masculinité " complice ${ }^{14}$ " chez les hommes juifs dans l'espace germanophone - p. ex. chez les étudiants ou les vétérans de guerre -, afin, par la masculinité, de rejoindre le cœur de la nation.

\section{Un corps masculin sous surveillance : discipline et contention}

Pour le (jeune) homme cherchant à être reconnu par ses 'pairs', l'apprentissage de la masculinité passait par le fait que le corps était constamment jaugé, scruté. Le corps masculin en apprentissage était sous surveillance permanente parce qu'il était au cœur même des préoccupations du groupe social. Josef Burg, né en 1909 à Dresde, se rappelle le contrôle social qu'il a subi en tant que jeune homme. Ce contrôle, qui succédait au contrôle familial, s'est exercé tout au long de sa vingtaine : il émanait du cercle amical, du mouvement de jeunesse " pionnier ", des camarades d'études. Il s'agissait à tout moment de jauger les agissements du jeune homme, afin qu'il se fonde dans la norme de sa classe sociale, de son sexe et de son identité juive. Le contrôle était d'autant plus intense que Josef était étudiant au séminaire rabbinique, dans un environnement juif orthodoxe. Sa vie sociale à Berlin se partageait entre le séminaire et l'université, distants d'un quart d'heure seulement. Il se souvient que chacun de ses mouvements était contrôlé ; on s'intéressait à l'heure à laquelle il se levait et allait se coucher, à ses allées et venues, aux personnes qui l'accompagnaient ${ }^{15}$.

13. George L. Mosse, L'Image de l’homme. L'invention de la virilité moderne [1996], Paris, Abbeville, 1997.

14. La sociologue Raewyn Connell pense le genre en lien avec d'autres rapports de domination, ce qui la conduit à une matrice de quatre types de masculinités. Il y a d'un côté la masculinité " hégémonique ", qui se trouve en position dominante dans une structure donnée de rapports de genre et qui est contestable (et historicisable). De l'autre, il y a les masculinités qui se définissent par rapport à elle : masculinités "subordonnées ", masculinités " complices ", masculinités " dominées ". Voir Raewyn Connell, Masculinités. Enjeux sociaux de l’hégémonie, éd. M. Hagège, A. Vuattoux, Paris, Éd. Amsterdam, 2014.

15. Israel-Korpus Emigrantendeutsch in Israel, $1^{\text {re }}$ génération (IK1), Entretien Miryam Du-nour avec Josef (Shlomo Yossef) Burg (né en 1909 à Dresde), Jérusalem, 27 janvier 1994. 
La socialisation à la masculinité passait aussi par l'identification d'attitudes et de zones du corps acceptables (et racontables). Apprendre à se comporter en homme, c'était apprendre à dominer ce corps, siège des émotions et réceptacle de la douleur. Les "incidents critiques " liés au corps, ceux où le cadre de la norme acceptable avait été enfreint, sont stockés dans une forme de mémoire corporelle et prennent une place significative dans les récits autobiographiques : il s'agit de donner un sens rétrospectif à l'incident, en le replaçant dans un contexte social plus large ${ }^{16}$. Pour les jeunes hommes juifs, l'apprentissage concernait le corps en général et ses postures, mais aussi, plus spécifiquement, le port de vêtements ou le soin apporté à la pilosité. Afin d'asseoir une identité masculine, il était important de " bien présenter " et de porter le cheveu court ${ }^{17}$. L'idéal bourgeois de discipline et de contention, calqué sur l'habitus militaire, exerçait de fortes contraintes sur l'apparence physique. Le corps masculin était tenu et retenu par des vêtements, qui influaient sur la gestuelle et la posture. C'est d'ailleurs ce code vestimentaire, ce port rigide et ces manières formelles que les hommes juifs originaires d'Allemagne (et d'Autriche) ont transféré au Proche-Orient et qui a été raillé, leur valant - selon l'une des étymologies possibles - le surnom de yekkes, i.e. " celui qui porte le veston " (yekke en yiddish).

La discipline et l'entraînement des corps préparant les hommes au combat, ou rappelant la situation de combat, permettaient d'" en imposer " et de s'imposer, de prendre possession de l'espace tout en domptant les affects. La pratique physique et sportive, marqueur d'appartenance sociale, était perçue comme un régulateur : elle permettait de distinguer les contacts acceptables de ceux qui ne l'étaient pas. Le récit de Gad (Gerhard) Beck, né en 1923 à Berlin, est particulièrement riche. Se remémorant les interactions avec les jeunes de son âge, il décrit ses deux amis gitans, les frères Herzberg, tous deux incarnant le " mauvais garçon ". Lui, le jeune homme issu de la bourgeoisie, qui est "à moitié juif " (il sera qualifié de "Mischling " dans le système racial nazi) et homosexuel, se sent particulièrement à l'aise avec ces outsiders. La gestuelle et le langage corporels des frères Herzberg ainsi que la proximité physique permise par leur éducation non bourgeoise exercent une attirance érotique sur le jeune Gerhard :

Ich hatte zwei Mitschüler, die Zigeuner waren, mit denen freundete ich mich besonders an. Sie wohnten fünfzehn Minuten von uns entfernt, in Berlins größter Zigeuner-Siedlung aus Wagen und Zelten. Die beiden Herzbergs waren ein paarmal sitzengeblieben und dementsprechend älter und stärker als ich ; sie gingen unregelmäßiger zur Schule, manchmal zogen sie auch eine Zeitlang mit ihrer Familie umher und lebten gar nicht in Berlin. Meine beiden " großen Freunde " beschützten mich, und ich bewunderte sie. Für sie hegte ich meine ersten Gefühle der Hingabe, ein Bedürfnis, mich anzukuscheln, denn sie stellten für mich

16. Voir Anke Abraham, Der Körper im biographischen Kontext, Wiesbaden, Westdeutscher Verlag, 2002. Voir aussi Eva-Maria Thüne, "Die Erzählungen des sprachlosen Leibs. Körpererfahrung und Identität im Erzählkorpus "Emigrantendeutsch in Israel” (1. Generation) ", in Arnulf Deppermann, Martin Hartung (dir.), Gesprochenes und Geschriebenes im Wandel der Zeit. Festschrift für Johannes Schwitalla, Mannheim, Verlag für Gesprächsforschung, 2013, p. 145-158.

17. Voir IK1, Entretien Kristine Hecker avec Franz Naphtali Krausz (né en 1905 à Sankt Pölten), Tel Aviv, $1^{\text {er }}$ octobre 1989. 
den Inbegriff von Männlichkeit dar. Ihre Körperlichkeit im Umgang miteinander kannte ich nicht, da fasste man sich viel an, täppisch und liebevoll mit knubbeligen, groben Händen ${ }^{18}$.

Pour les jeunes hommes juifs, la conformité à ces injonctions genrées était l'un des enjeux de l'assimilation à la classe sociale dominante : la bourgeoisie. Les comportements " adaptés " étaient inculqués dans ces fabriques de la masculinité qu'étaient l'école, la rue ou la cour de récréation. Toujours dans une perspective détournée par l'expression d'une subjectivité homosexuelle, Gad Beck se remémore les cours et exercices de sport, censés développer la cohésion de groupe, la coordination du corps et de l'esprit, et donc une masculinité assurée :

Dazu kam, nicht zu vergessen, der Sport ! Ich war darin nicht schlecht, aber für mich stand dabei das Erotische im Vordergrund. Es gab eine Übung, bei der alle Jungen in einer Reihe hintereinander standen, und der Hinterste musste mit dem Ball zwischen den gespreizten Beinen der anderen hindurchkriechen bis nach vorn, dann der nächste ; na, das waren Aussichten ! Oder wenn man an einer Stange hochkletterte und wieder runterrutschte - die Hälfte der Jungs hatte nachher nasse Turnhosen von der Reibung. Das genoss ich wie heute manche Leute Pornofilme ${ }^{19}$.

\section{L'antisémitisme comme atteinte au corps viril}

Lorsque deux officiers de la SS sont venus chercher le père de Benjamin Kedar, né en 1923, ce dernier se souvient ne pas avoir pleuré (" nous n'étions pas éduqués à pleurer ") :

Mein Vater war Oberamtsgerichtsrat. Er wurde verhaftet [...] und das war für mich ein großer Schlag. Also ich hab meinen Vater immer sehr verehrt, aber wahrscheinlich, ohne dass ich mir darüber klar war. Und auf einmal wurde er abgeholt von zwei SS-Leuten. Daran kann ich mich gut erinnern. Meine Mutter hat uns erst weggeschickt, woanders spielen, und dann, auf einmal hat sie uns zurückgerufen. Wahrscheinlich hat sie gedacht, wenn wir ein bisschen weinen, dass das diese Männer rührt. Wir waren aber nicht erzogen zu weinen und mein Vater war auf einmal weg ${ }^{20}$.

En se comportant " en bon petit soldat " ce jour-là, en ne pleurant pas pour essayer d'attendrir les officiers, Benjamin aurait contribué à l'arrestation du père. Cette culpabilité, liée ici à une socialisation particulière à la masculinité, transparaît encore dans le récit près de soixante ans plus tard.

Se comporter en homme " allemand " signifiait se conformer au modèle hégémonique, et toute divergence devenait stigmatisante. Dans son autobiographie, Schlomo Rülf, né en 1896, se rappelle la position "à part " qu'il avait à l'école : il était juif, marqué par une apparence physique " exotisée " - la couleur de cheveux p. ex. -, mais son rapport à l'éducation (de Bildungsbürger) et son jeune

18. Frank Heibert (éd.), Und Gad ging zu David. Die Erinnerungen des Gad Beck 1923 bis 1945, Berlin, Edition diá, 1995, p. 19.

19. Ibid., p. 31.

20. IK1, Entretien Miryam Du-nour avec Benjamin Kedar (Benjamin Kopfstein, né en 1923 à Seesen), Jérusalem, 12 novembre 1993. 
âge l'empêchaient également d'accéder à une socialisation pleinement virile faite de coups, de corps-à-corps et de résistance à la douleur. En filigrane, son récit introduit la possibilité d'une forme alternative d'être un garçon : une masculinité reposant sur l'instruction, la culture et la non-violence. Son récit incorpore aussi des stéréotypes antisémites et rappelle l'existence d'une " frontière invisible " raciale et genrée, intériorisée par tous et donc infranchissable : une "unüberbrückbare Kluft ${ }^{21}$ ". L'antisémitisme " ordinaire " reposait sur la stigmatisation des corps. Il n'impliquait pas nécessairement la violence ${ }^{22}$ et Schlomo Rülf se rappelle avoir plutôt joui d'une relative tranquillité dans sa jeunesse, en comparaison avec les violences ultérieures :

Denn ich war sehr verschieden von den blonden, derben Jungen meiner Klasse, die der kräftigen niedersächsischen Rasse angehörten und rauhen Sitten huldigten. Sie veranstalteten untereinander allerlei Wettkämpfe und Prügeleien, aber mich, den weitaus Jüngsten der Klasse, ließen sie meist in Ruhe. Ich war wohl in ihren Augen ein feines exotisches Gewächs, das man behutsam anfassen musste. [...] Ich sprach ein gewähltes Deutsch, schrieb einen guten Aufsatz und gehörte zu den guten Schülern, obgleich ich mich nicht besonders anstrengte ${ }^{23}$.

Pour Ephraïm Orni (Fritz Schwarzbaum), né en 1915, c'est moins au sein de la cour d'école que dans la rue que se manifestait la stigmatisation. Comme dans l'exemple précédent, le jeune Fritz était visé à plusieurs titres. En tant que garçon juif et en tant que garçon légèrement handicapé :

\footnotetext{
In dieser Schule war die große Mehrheit der Schüler jüdisch, so dass ich damals praktisch keinen Antisemitismus in der Schule verspürte. Wenn ich ihn doch verspürte, so beim Spielen auf der Straße. Wir wohnten in einer Gegend, wo nur eine sehr geringe Zahl von Juden wohnten und die meisten Kinder waren eben damals auch schon antisemitisch erzogen und wiesen mich gerne ab. Schon deswegen, weil ich damals schon körperlich leicht behindert war. [...] Die Bezeichnung Jude galt schon bei vielen Kindern als Schimpfwort und das bekam ich zu spüren ${ }^{24}$.
}

Le corps juif masculin apparaît comme une surface de projection, d'expérimentation et de mise en scène des représentations raciales, sociales et genrées. Il fait l'objet d'une négociation concernant l'appartenance au corps social national. Le pouvoir et les formes de domination s'incarnent dans les injonctions normatives faites aux corps. Dans les interactions quotidiennes, un garçon ou un (jeune) homme juifs étaient régulièrement ramenés à leur corporalité, et leur masculinité sans cesse remise en jeu. Ainsi, le jeune Gerhard Beck est-il stigmatisé pour son odeur corporelle supposée. Au cours de l'année 1933, un camarade de classe lance

21. Schlomo Rülf, Ströme im dürren Land. Erinnerungen, Stuttgart, DVA, 1964, p. 20.

22. Micha Michaelis en revanche se souvient des flambées de violence dont il fut victime enfant, en pleine guerre. À l'issue d'un passage à tabac, il fut transporté à l'hôpital pour traumatisme crânien. IK1, Entretien Anne Betten avec Micha Michaelis (né en 1908 à Berlin), Kibboutz Dalia, 30 avril 1991.

23. Rülf, Ströme im dürren Land, p. 15.

24. IK1, Entretien Anne Betten avec Ephraïm Orni (Fritz Schwarzbaum, né en 1915 à Breslau), Jérusalem, 20 avril 1991. 
soudain que Gerhard pue le pied juif transpirant ${ }^{25}$. Parfois, l'injure se transforme en simple " Juif qui pue " (Judenstinker ${ }^{26}$ ).

C'est par le biais de l'identité virile, et par le biais d'un vécu corporel que les jeunes gens juifs en Allemagne et en Autriche ont perçu l'antisémitisme dont ils étaient la cible. Dans son histoire de la vie juive sous le nazisme, Marion Kaplan rappelle que si les Juifs furent bien la cible d'attaques 'en tant que' juifs, les modalités de l'antisémitisme étaient éminemment genrées. Ce sont les hommes qui furent les toutes premières victimes de l'antisémitisme radicalisé des années 1930, qu'il s'agisse d'attaques physiques subies et d'arrestations, ou encore de mesures discriminatoires les privant d'identité professionnelle et sociale ${ }^{27}$. Parce qu'ils avaient davantage accès à l'espace public, les mesures antisémites discriminatoires les ont immédiatement affectés. Marion Kaplan souligne également à quel point la montée de l'antisémitisme était difficile à vivre pour les jeunes, qui n'étaient pas en mesure de donner un sens à la discrimination dont ils étaient les victimes de la part des autres ${ }^{28}$.

Dans les récits rétrospectifs de Juifs allemands, et de manière peut-être plus dramatique encore dans les récits de Juifs autrichiens après 1938, une place importante est faite aux épisodes relatant l'atteinte portée à la liberté de mouvement et à l'usage de l'espace public. Ces épisodes contrastent douloureusement avec une socialisation à la masculinité où les hommes étaient éduqués à jouir d'une relative liberté de mouvement. Gawriel Walter, né en 1921 à Kolberg (Poméranie), se souvient avec émotion de la première fois où l'entrée de la piscine lui fut refusée. Un écriteau portait la mention « Interdit aux Juifs et aux chiens " :

Ich glaube eine Sache, die mich noch jahrelang verfolgt hat, war, ich habe angefangen Schwimmen zu lernen und wie ich dann in die Kolberger Badeanstalt kam und dort ein Schild vorfand, "Juden und Hunde unerwünscht ", das hat mir einen furchtbaren Schock verursacht ${ }^{29}$.

Interrogé sur la montée de l'antisémitisme, Abraham Goldberg, né en 1923 à Altenburg (Thuringe), convoque ses souvenirs de garçon de dix ans. Il se souvient des lieux publics devenus inaccessibles et de la blessure ressentie. Dénoncé par un camarade de classe, il ne peut plus fréquenter le cinéma, interdit " aux Juifs et aux chiens ", pour y voir le film pour enfants du dimanche. Cette première restriction de sa liberté d'aller et venir n'est rien en comparaison de l'interdiction de fréquenter la piscine, alors qu'il s’y entraîne tous les après-midis afin, justement, de fortifier son corps d'homme en devenir :

25. Heibert (éd.), Und Gad ging zu David, p. 25.

26. IK1, Entretien Kristine Hecker avec Isack Bayer (né en 1902 à Aschbach), Jérusalem, 5-6 juillet 1990.

27. Marion A. Kaplan, Between Dignity and Despair. Jewish Life in Nazi Germany, New York/ Oxford, Oxford UP, 1998.

28. Ibid., p. 107.

29. IK1, Entretien Anne Betten avec Gawriel (Herbert, né en 1921 à Kolberg, Poméranie) et Käthe Walter (née en 1925 à Katscher, Haute-Silésie), Sde Warburg, 28 avril 1991. 
Ich konnte nicht mehr ins Kino gehen. Aber das war noch nicht so schlimm wie das Schwimmen. Ich schwimme sehr gerne, auch heute noch. Und es gab natürlich ein großes Schwimmbad, ein städtisches Schwimmbad in Altenburg. Ich war Freischwimmer und ich bin im Sommer jeden Nachmittag schwimmen gegangen, bis eines schönen Tages ein großes Schild war : "Juden und Hunden Zutritt verboten ». Erstens weiß ich nicht, warum die Juden mit den Hunden immer zusammengingen, denn schließlich haben die Leute die Hunde gerne gehabt, das ging aber zusammen, Juden und Hunde. Und ich komm nach Hause, hab gesagt, was, schwimmen darf ich auch nicht mehr, ich kann nicht mehr baden gehen ${ }^{30}$.

Son père a l'idée de le conduire le dimanche dans d'autres piscines de la région, où on ne le connaît pas. Cela ne dure qu'un temps et bientôt, l'accès des piscines alentours lui est interdit. Sa capacité de mouvement dans l'espace public est réduite, le jeune Abraham ne peut plus exercer son sport favori : il ressent pleinement la distance qui le sépare du vécu des autres garçons. Brutalement, parfois du jour au lendemain, les pratiques de l'espace étaient modifiées. À l'école, dans les classes ou la cour, une place à part était désormais assignée aux Juifs. Abraham Frank se souvient avoir été contraint de s'asseoir dans les derniers rangs de la classe, à la place des cancres, et avoir eu interdiction de courir pendant les récréations : assigné au coin, il ne pouvait ni jouer ni converser avec les autres ${ }^{31}$.

Si le corps juif masculin était stigmatisé, il recevait également des coups. Il ne s'agissait plus simplement des rudoiements faisant le quotidien des garçons. Les coups étaient d'un autre ordre : il s'agissait d'une violence de groupe visant à faire mal, à blesser. Abraham Goldberg rappelle qu'il a " pris des coups ", que " tout un groupe s'est jeté sur [lui] ":

Da war eine Gruppe von fünf, sechs Jungens, die, wenn ich rausgegangen bin von zu Hause, mir nachgegangen sind, und sie haben mich überfallen, haben mich geschlagen. [...] Aber es sind immer vier, fünf über mich hergefallen in der Pause und man hat mich, ich kann mich erinnern, man hat mich manchmal mit Strick gebunden, die Hände und Füße, und man hat mich so geworfen ${ }^{32}$.

C'est là le récit courant de l'antisémitisme ordinaire lorsque l'on était un adolescent juif. Le poète israélien Yehuda Amichaï, né en 1924 à Würzburg, se souvient des pierres reçues sur le chemin de l'école, l'obligeant à emprunter des chemins détournés ${ }^{33}$; Ernst Schwarz (né en 1913) souligne les injures et les crachats dont il fut victime ${ }^{34}$.

Parce que l'antisémitisme était un " code culturel $^{35}$ " partagé, parce qu'il faisait système, les garants et les figures d'autorité étaient défaillantes et les jeunes Juifs se retrouvaient livrés à eux-mêmes. Dans les souvenirs des enfants

30. IK1, Entretien Kristine Hecker avec Abraham Goldberg, Ramat Gan, 4 juillet 1990.

31. Abraham Frank, "Lebens-Geschichte ", [http://www.irgun-jeckes.org/?CategoryID=345\&tArticle ID=747], dernière consultation le 30 septembre 2015.

32. IK1, Entretien Kristine Hecker avec Abraham Goldberg, Ramat Gan, 4 juillet 1990.

33. IK1, Entretien Miryam Du-nour avec Yehuda Amichaï (Ludwig Pfeuffer, né en 1924 à Würzburg), Jérusalem, 23 janvier 1994.

34. IK1, Entretien Miryam Du-nour avec Ernst Schwarz (né en 1913 à Cham, Haut-Palatinat), Bar Ilan, 11 mai 1993.

35. Shulamit Volkov, Antisemitismus als kultureller Code. Zehn Essays, Munich, C. H. Beck, 2000. 
et jeunes hommes, rares sont les récits d'interventions protectrices. Les parents, eux-mêmes visés de plein fouet, étaient impuissants. Et les maîtres, éducateurs ou surveillants se rangaient souvent du côté du groupe majoritaire. Le récit de Gershon Monar, né en 1924 à Leipzig, fait exception. Il y est question d'un maître, " pourtant plutôt nazi ", intervenu pour rappeler qu'il n'était pas héroïque de se mettre à plusieurs contre un :

Ich kann mich noch so dunkel an die Schulzeit erinnern und dass da auch schon so ein gewisser Antisemitismus in der Klasse war. Ich habe auf dem Nachhauseweg hier und da Prügel bekommen und weiß eigentlich nicht genau warum. Einer der Lehrer, der sogar eigentlich ein ziemlicher Nazi war, der hat dann auch gesagt, dass das eigentlich keine große Heldentat wäre, wenn die dreißig Schüler sich auf den einen Juden stürzen ${ }^{36}$.

L'antisémitisme quotidien se présentait sous la forme d'une pression exercée par le groupe majoritaire. Il était vécu à même le corps, qui encaissait les coups sans pouvoir les rendre, ce qu'aurait pourtant dicté le code de conduite viril.

En retour, ce corps incapable de se défendre se voyait « féminisé ». Dans le récit de vie de Heinrich Mendelssohn, né en 1910 à Berlin, la prise de conscience de l'antisémitisme ambiant est associée à des souvenirs de " rixes ", de " crochets " et de " coups " dans la cour de l'école. Le narrateur insiste sur la prise de conscience de la frontière invisible qui, à l'école, séparait les outsiders - en l'occurrence le " Juif ", l'" Italien " et le " Bulgare " - du reste du groupe :

Ich freundete mich da mit zwei Minoritäten an. Ein Italiener, dessen Vater Italiener war, die Mutter Deutsche, und ein Bulgare, dessen Vater Bulgare war und die Mutter Deutsche, und mit denen waren wir also ein Minoritätentrio ${ }^{37}$ !

Il se souvient également n'avoir pas fait preuve d'un comportement suffisamment viril à l'époque en réaction aux provocations :

Sie sagten : "Ach, die Juden sind doch alle Betrüger, sind doch alle Gauner " und so weiter, nicht? Also es fing eine Prügelei an und es fielen mehrere über mich her und ich war sehr unterlegen gegen die, und außer Boxen und Schlagen habe ich dann auch gekratzt. Da waren sie sehr beleidigt und liefen zum Lehrer und haben mich verpetzt : " Der Mendelssohn hat gekratzt ". Sag ich : " Ja, aber viele gegen einen, das gehört sich nicht ". Sagt der Lehrer : " Ja, das ist richtig, das gehört sich nicht, aber du darfst auch nicht kratzen ». Und das fand ich ungerecht, da sah ich das erste Mal, was Antisemitismus ist (LACHT) ${ }^{38}$.

L'anecdote se clôt sur un rire rétrospectif, qui est la trace narrative du traumatisme subi. Heinrich Mendelssohn établit un lien entre l'injure antisémite et la stigmatisation genrée de ne pas s'être comporté " en homme " (parce qu'il a griffé pour se défendre).

Se remémorant l'année 1934, Hans Simon Forst évoque quant à lui la frustration et la douleur de ne pas avoir pu riposter " en homme ". Il s'agit là,

36. IK1, Entretien Anne Betten avec Gershon Monar (Günter Albert Mundstock, né en 1924 à Leipzig), Haïfa, 29 avril 1991.

37. IK1, Entretien Anne Betten avec Heinrich Mendelssohn (né en 1910 à Berlin), Tel Aviv, 25 avril 1991.

38. Ibid. 
par le biais de la stigmatisation antisémite, d'une blessure profonde de l'identité de genre :

Eines Tages bin ich auf der Straße angerempelt worden und konnte mich nicht wehren als Judenjunge, zusammen mit anderen Juden, die auch angerempelt wurden von einer Schar SA. Es war sehr unangenehm, denn ich war gewöhnt mich zu wehren, wenn ich angerempelt wurde und das war gegen vier, fünf große Schläger, da konnte ich nichts machen, ich bin weggelaufen, und das ist mir so zu Herzen gegangen ${ }^{39}$.

Dans le contexte de la flambée d'antisémitisme, il était douloureux de 'ne plus' être perçu par les autres comme un corps masculin en mesure de se défendre. Ne pas être " bousculé ", " être de l'air ", représentait une blessure profonde de l'identité virile. Hans Simon Forst poursuit :

Aber wie ich nach Hause fuhr zu den Feiertagen, hatte ich ein sehr unangenehmes Erlebnis, denn unterwegs stiegen Jungens in den Zug ein, die ich von der Schule aus kannte, frühere Klassenkameraden. Und ich war für sie Luft ! Sie haben mich nicht angerempelt, haben nicht mit mir gesprochen. Es war ein sehr unangenehmes Gefühl, man hat stundenlang zusammengesessen in einem Coupé. Das war sehr unangenehm. Ich habe zum Fenster rausgekuckt, mir die Gegend angesehen, das war alles ${ }^{40}$.

Cette anecdote l'a profondément marqué. Alors qu'il est interviewé quelques années plus tard, il relate les mêmes faits, utilisant des formulations identiques : " j'étais de l'air pour eux, ils ne m’ont pas remarqué, ne m’ont porté aucune attention $^{41}$ ".

Évoquant le rejet antisémite dont il fut l'objet, Moshe Ballorn, né en 1913 à Berlin, émaille son récit de métaphores corporelles signalant une dévirilisation :

Da ist wieder der Unterschied zwischen deutschen Juden und den Zugereisten, wissen Sie, oder den Nachkommen. Unsere Kinder, sagen wir mal, das waren nie deutsche Juden, die sind nicht in Deutschland zur Schule gegangen, die haben das Deutschtum nicht mit Löffeln gefressen, verstehen Sie und nachher einen Tritt in den Hintern gekriegt, die haben das niemals erlebt. Die haben niemals dieses tiefe seelische Erlebnis gehabt, ausgestoßen zu werden, rausgeschmissen zu werden aus ihrer Heimat ${ }^{42}$.

C'est bien une blessure intime qui s'exprime, une blessure du sentiment d'être un homme. L'exclusion antisémite s'incarne métaphoriquement par le " coup de pied dans le derrière ", zone par excellence de la dévirilisation. Par ailleurs, le derrière est la zone même du " rejet ", de l'" expulsion " (le mot " ausstoßen " est plus fort encore).

Il a été question jusqu'ici de formes de discrimination plus ou moins virulentes, impliquant coups, brimades, jet de projectiles et ostracisation. Toutes visaient à la fois le Juif et le (jeune) homme et mettaient en jeu la capacité

39. IK1, Entretien Eva Eylon avec Hans Simon Forst (né en 1917 à Kastellaun), Tel Aviv, 25 novembre 1991.

40. Ibid.

41. IK1, Entretien Anne Betten avec Hans Simon Forst (né en 1917 à Kastellaun), Eichstätt, 19 mai 1994.

42. IK1, Entretien Anne Betten avec Moshe (Max) Ballhorn (né à Berlin en 1913), Tibériade, 1er juillet 1990. 
d'un corps masculin à se défendre et à s'épanouir dans l'espace public. Mais la radicalisation rapide de l'antisémitisme après 1933 a poussé la logique plus loin encore : de l'ostracisation et de la blessure morale, on est passé aux blessures physiques et à l'humiliation infligées par la SA. Si l'anecdote de la griffure prête éventuellement à sourire, les menaces dont fut victime Heinrich Mendelssohn plus tard, durant ses études à la Charité de Berlin, étaient beaucoup plus sérieuses. En février-mars 1933, après une altercation au sujet d'un siège dans un amphithéâtre bondé, le jeune Heinrich est attendu par une bande de SA : il se réfugie en bibliothèque jusqu'au soir. Cette expérience est convoquée pour expliquer directement l'émigration en Palestine en juillet 1933. Dans l'intervalle, interrompant ses études de médecine, Mendelssohn propose ses services à la polyclinique juive de la Bülowplatz à Berlin. Il y est témoin de la brutalité nazie, et soigne des corps d'homme dénudés, humiliés, couverts de blessures et d'ecchymoses ${ }^{43}$.

Les récits autrichiens font apparaître à quel rythme l'antisémitisme s'est radicalisé. Si les violences antisémites n'ont pas commencé avec l'entrée des troupes allemandes le 12 mars 1938, le choc ressenti par les quelque 190000 Juifs autrichiens fut particulièrement brutal. Le processus de déchéance des droits, d'ostracisation et d'exclusion progressive, qui avait pris cinq ans en Allemagne, a eu lieu en quelques mois. En plus des mesures d'exclusion du marché du travail et de l'espace public (parcs et jardins, piscines, berges du Danube), les témoignages autrichiens évoquent des mesures d'humiliation répétées dans un climat de peur : les Juifs étaient frappés publiquement, contraints de s'agenouiller et de nettoyer les trottoirs, de marcher en cercle jusqu'à l'épuisement, de manger de l'herbe. L'historien israélien Shaul Baumann, né en 1922 à Vienne, se souvient de ces quelques mois entre l'annexion de l'Autriche par l'Allemagne nazie et son émigration en Palestine en 1939 :

\begin{abstract}
Diese Aktionen mit den Zahnbürsteln, wo man die Juden aus der Wohnung rausgeholt hat und die Trottoire mit Zahnbürsteln reinigen hat lassen oder so, die Juden schikanieren, dann hat das angefangen, nicht? Da wusste man schon, es wird gefährlich. Und dann kamen nach und nach die Gesetze : Juden dürfen nicht in die öffentlichen Anlagen gehen, dürfen sich nicht auf ein Bankl setzen, da darf man nicht einkaufen, und da darf man nicht, das darf man nicht, und dies nicht. Da gab's allerhand Schikanierungen ${ }^{44}$.
\end{abstract}

Paul Feiner, né en 1919, a été victime de ces mesures d'humiliation. À l'été 1938, quelques semaines seulement avant son émigration, il est arrêté en pleine rue dans le $2^{\mathrm{e}}$ arrondissement, où vivent de nombreux Juifs. Il est contraint de se mettre à genoux et de nettoyer la rue puis le sol d'un commerce. La posture corporelle et la tâche à accomplir visent à humilier le Juif en tant qu'homme et à le " rabaisser " en le "féminisant ". L'opération se termine par un coup de pied

43. IK1, Entretien Anne Betten avec Heinrich Mendelssohn (né en 1910 à Berlin), Tel Aviv, 25 avril 1991.

44. IK1, Entretien Margareta Strasser et Clemens Nirnberger avec (Otto) Shaul Baumann (né en 1922 à Vienne), Jérusalem, 30 novembre 1998. 
dans le derrière, qui vise cette fois-ci le jeune homme de dix-neuf ans, quasiment adulte et pourtant, par ce geste même, traité en petit garçon ${ }^{45}$.

\title{
Conclusion : récits de riposte
}

En réaction à un environnement où l'antisémitisme envahit la sphère intime et corporelle, l'une des réponses juives fut l'instauration de formes de riposte - dans la rue, à l'école, au sein d'associations sportives, dans les organisations de jeunesse. Cette réaction, qui prolongeait un discours sioniste de régénération par le " Nouveau Juif " et le " judaïsme du muscle ", passait par l'aptitude à se défendre ou, au moins, à adopter une posture de riposte. D'une manière paradoxale, les valeurs de l'homme sioniste se tuilent donc avec les valeurs martiales (et " allemandes ") de la bourgeoisie ${ }^{46}$. Il n'est pas étonnant que le langage, les codes, les rites et les postures corporelles aient été partagés, du moins en partie, même avec les mouvements d'extrême-droite. Iwan Lilienfeld, né en 1910 à Ryknik (Haute-Silésie) s'en émeut rétrospectivement :

\begin{abstract}
Aber heute kann man sich nur wundern und über sich selber den Kopf schütteln, wie wenig man die Vorzeichen zur Kenntnis genommen hat und daraus die Folgerungen gezogen hat. Wenn ich mir heute vorstelle, dass wir in der Jugendbewegung gar nichts daran fanden, uns mit Heil zu begrüßen und Lagerfeuer zu veranstalten. Und das ganze Vokabular, das später die Nazis übernommen hatten ! Da muss ich mir heut nur an den Kopf fassen ${ }^{47}$ !
\end{abstract}

Cette ambiguïté a donné naissance à des continuités existentielles et émotionnelles étonnantes, comme en témoignent les changements d'affiliation relatés au fil des récits. Cela a également conduit à solidifier un répertoire culturel, corporel et genré transcendant les clivages idéologiques. Micha (Fritz) Michaelis, né en 1908, raconte sa rencontre au milieu des années 1930 avec l'un de ses anciens camarades, plus âgé que lui et qui l'avait fait entrer dans le mouvement de jeunesse Alt-Wandervogel. Le camarade en question porte l'uniforme de la SS. Or le frère de Micha, juif et communiste, vient d'être arrêté et envoyé à Oranienburg :

Und er sprach mich an auf der Straße und sagte, was ist los mit dir, ich kenn dich doch gar nicht mit dem Kopf nach unten. Und ich guckte ihn an und er war in SS-Uniform, und ich sagte, du hast wohl vergessen, dass ich Jude bin ${ }^{48}$.

Transcendant le contexte politique et les clivages de l'époque, les deux hommes continuent de partager un code de conduite masculin. Dans leur échange, il

45. IK1, Entretien Anne Betten avec Paul Feiner (né en 1919 à Vienne), Haïfa, 27 juin 1990.

46. Jehuda Reinharz souligne la " consonance " idéologique fondamentale du sionisme avec le milieu dont il est issu, la bourgeoisie. Jehuda Reinharz, "Ideology and Structure in German Zionism, 1882-1933 ", in J. Reinharz, Anita Shapira (dir.), Essential Papers on Zionism, New York, NYUP, 1996, p. 287.

47. IK1, Entretien Anne Betten avec Iwan Lilienfeld (né en 1910 à Rybnik, Haute-Silésie), Ramat Gan, 26 avril 1994.

48. IK1, Entretien Anne Betten avec Micha (Fritz) Michaelis (né en 1908 à Berlin), Kibboutz Dalia, 30 avril 1991. 
y a le rappel à l'injonction genrée à se comporter en homme, à adopter la posture et la disposition d'esprit apprises dans la jeunesse - qui constituent un répertoire culturel commun. Moshe Ballhorn est l'un des rares à expliciter le lien de continuité entre les valeurs national(ist)es allemandes et celles du sionisme. Il rappelle que son engagement d'adolescent en faveur de l'Allemagne était 'de même nature' que son engagement pour les valeurs " juives-nationales " :

Im Jahre 1921 hat mein Vater, der völlig assimiliert war, aber ein bewusster Jude, der hat uns Jungens in den Deutschen Turnverein geschickt. Da waren wir dann durch meine ganze Schulzeit, wir waren befreit von Körperübungen in der Schule, weil wir im Deutschen Turnverein waren. Wir waren gute Turner und dieser Turnverein hat mich geprägt, der hat mich so geprägt, dass ich mich heute noch als Preuße fühle, ich kann nichts dafür ! (LACHT) [...] Da war ich vierzehn Jahre alt und ich war Hundertprozent Deutscher. [...] Da hab ich auf einmal gemerkt, halt da stimmt was nicht. [...] Und ich wollte so, wie ich deutsch-national eingestellt war, zu hundert Prozent, so wollte ich jüdisch-national sein und bin dann Zionist geworden ${ }^{49}$.

Moshe Ballhorn rejoint le mouvement sioniste révisionniste du Betar (ou Brith Trumpeldor ${ }^{50}$ ), qui l'envoie dès 1932 à Günzdorf pour y suivre une préparation paramilitaire au départ en Eretz Israël. Il y apprend à marcher au pas et suit un entraînement militaire. Grâce à la socialisation au sein d'une association gymnique, tout cela ne lui est pas étranger. Pour lui, la « conversion " au sionisme s'est faite dans la continuité : dans la posture, dans la disposition d'esprit et par l'intermédiaire de pratiques corporelles.

Cette " conversion " au sionisme était un processus comportant des degrés et des moments-charnières. Le sionisme, ses organisations et leurs rites apparaissent rétrospectivement à certains comme les avatars ultimes d'une culture nationaliste envers laquelle ils ont des sentiments ambigus. D’une part, ils ont conscience du fait que la culture sioniste, juive-nationale, a exacerbé les valeurs militaristes, belliqueuses et virilistes. D'autre part, ces mêmes rites ont fourni des éléments de continuité émotionnelle et identitaire dans un parcours de vie marqué par la rupture migratoire. Les permanences de l'identité de genre ainsi construite ont permis à certains de s'" y " retrouver, par-delà les aléas de la vie. Rétrospectivement, cette conversion au sionisme a parfois permis de prendre une revanche historique. Micha Michaelis construit le récit de son premier retour en Allemagne en 1959 comme la revanche du "Juif musclé " qui ne se laisse plus insulter. Alors qu'il se promène à Essen dans l'artère commerçante en quête d'une adresse, il heurte un passant :

Und dann hat der Mann gesagt : " Du verfluchter Jude, dich hat man auch vergessen, in Auschwitz zu verbrennen ! " Und dann habe ich als Waffe - ich habe mal einen Jiu-JitzuKurs in meiner Jugend mitgemacht. Und eine phantastische Waffe ist ein Hausschlüssel, der vorne spitz ist, mit dem man also schlagen kann. Und den hatte ich mitgenommen, den hab ich aus der Tasche gezogen und hab ihn geschlagen, er hat zurückgeschlagen und

49. IK1, Entretien Anne Betten avec Moshe (Max) Ballhorn, Tibériade, 1er juillet 1990.

50. Betar est l'acronyme de Brith (Alliance) Trumpeldor, mouvement de jeunesse radical fondé en 1923 à Riga et proche du sionisme révisionniste incarné par Vladimir Zeev Jabotinsky. Ce nom rappelle à la fois Yoseph Trumpeldor, héros du sionisme contemporain, et la forteresse de Betar, lieu de la résistance des Juifs contre l'Empire romain au $\mathrm{II}^{\mathrm{e}}$ siècle. 
schließlich lagen wir beide auf der Straße und haben uns gegenseitig geschlagen. Und dann kam natürlich die Polizei und hat uns verhaftet. Er blutete und ich hatte ihm eine Rippe angeknackt ${ }^{51}$.

Dans les narrations autobiographiques et les récits de vie, qui portent les traces matérielles de l'injure raciale et genrée, ce sont des identités blessées qui se racontent a posteriori. Le récit fait une place aux stigmates incorporés, c'est-à-dire aux traces laissées par la violence antisémite. Certes, le récit ne donne qu'un aperçu de la manière dont les corps ont été affectés par l'injure et la stigmatisation... mais il en a néanmoins gardé la trace narrative tangible, matérielle.

51. IC1, Entretien Anne Betten avec Micha (Fritz) Michaelis (né en 1908 à Berlin), Kibboutz Dalia, 30 avril 1991. 
